\title{
Controlled Clinical Trial on Bone-anchored Hearing Implants and a Surgical Technique With Soft-tissue Preservation
}

\author{
Christine A. den Besten, Arjan J. Bosman, Rik C. Nelissen, Emmanuel A. M. Mylanus, \\ and Myrthe K. S. Hol
}

Department of Otorhinolaryngology, Radboud University Medical Centre, Nijmegen, The Netherlands

\begin{abstract}
Objective: To compare the clinical and audiological outcomes after linear incision with soft-tissue preservation and standard linear incision with soft-tissue reduction for placement of percutaneous bone-anchored hearing implants.

Study Design: Clinical trial with historical control-group from a previous randomized controlled trial.

Setting: Tertiary referral center.

Patients and Interventions: Twenty-five patients were enrolled in a prospective cohort of bone-anchored hearing implant placement with linear incision and tissue preservation with a follow-up of 6 months. The control-group consisted of 25 patients from a previous randomized controlled trial in the same tertiary referral center. All sound processors were fitted 3 weeks after surgery.

Main Outcome Measures: Numbness around the abutment, length of surgery, soft-tissue reactions according to Holgers' classification, Patient and Observer Scar Assessment Scale, implant loss, Implant Stability Quotient, and audiological outcome.

Results: Tissue preservation resulted in better results on sensibility (mean percentage correct responses 98\% [SD 4.4]
\end{abstract}

versus $89 \%$ [SD 15.0], $p=0.003)$, on the Patient and Observer Scar Assessment Scale (mean observer score 15.3 [SD 4.3] versus 19.4 [SD 6.3], $p=0.006)$, and shorter total surgery time (mean 24.6 min [SD 6.2] versus 31.9 min [SD 6.5], $p<0.001)$. More adverse soft-tissue reactions as measured by the Holgers classification were observed in the test-group $(\mathrm{n}=7$ [28\%] versus $\mathrm{n}=1[4 \%], p=0.049)$. For Implant Stability Quotient and audiology the study did not provide evidence that tissue preservation is better or worse compared with tissue reduction.

Conclusion: Tissue preservation compared with tissue reduction leads to a generally favorable clinical outcome, comparable audiology results, and significantly shorter surgery time. Longer follow-up is warranted to conclude on the increased adverse soft-tissue reactions after 6 months. Key Words: Baha-Bone-anchored hearing-Bone conduction-Hearing loss-Holgers-Implant loss-Soft tissue reactions-Tissue preservation.

Otol Neurotol 37:504-512, 2016.
The surgical procedure for percutaneous titanium implants for bone conduction hearing has been modified

Address correspondence and reprint requests to Christine A. den Besten, M.D., Department of Otorhinolaryngology, Radboud University Medical Centre, Post 377, PO box 9101, 6500 HB Nijmegen, The Netherlands; E-mail: Chrisje.denBesten@radboudumc.nl

Oticon Medical AB provided financial support for conducting the current study. In collaboration with all authors, Oticon Medical, designed the study, were involved in data interpretation and editing of the report. Data were recorded at the clinical center and monitored by Oticon Medical. Data management and statistical analysis were performed by external data managers and biostatisticians. All authors had full access to the data. The authors had final responsibility for the decision to submit for publication.

All authors report financial support to the authors' institution for conducting two clinical studies from Oticon Medical AB (Askim, Sweden) and from Cochlear Bone Anchored Solutions AB (Mölnlycke, Sweden), outside the submitted work.

This is an open access article distributed under the Creative Commons Attribution-NonCommercial-NoDerivatives License 4.0, where it is permissible to download, share and reproduce the work in any medium, provided it is properly cited. The work cannot be changed in any way or used commercially. several times since its introduction. During the last two decades there has been a tendency to less invasive surgery. When reviewing several frequently used techniques, the linear incision has been shown to be superior in several studies $(1-3)$. With the linear incision technique, as well as new implant and abutment designs, complications such as implant loss and adverse soft-tissue reactions have decreased to 0 to $4 \%$ and 8 to $15 \%$ respectively per implant in the adult population $(4,5)$.

In 2011, Hultcrantz (6) described a modification of the linear technique, without soft-tissue reduction. The rationale of soft-tissue preservation is less scar tissue formation, resulting in less numbness, cosmetic advantages, and faster wound healing. Furthermore, tissue preservation results in shorter surgery times. This technique was shown superior on these matters in this study and consequently the preservation technique was adopted by many surgeons. However, most comparative studies use no or a less ideal control-group, including dermatome technique, or test-groups with a variation on the preservation technique, like a (modified) punch 
technique (6-13). Additionally, audiological outcomes have not been reported thus far, whereas it has been mentioned that leaving the subcutaneous tissue around the abutment might dampen the vibrations to the skull (14).

The objective of the current study was to compare the aforementioned clinical and audiological outcomes uniformly after linear incision with soft-tissue preservation and a standard linear incision with soft-tissue reduction.

\section{METHODS}

\section{Study Design and Participants}

The current study was set up as a clinical trial on soft-tissue preservation with a historical control-group in which soft-tissue reduction was applied. Twenty-five patients were consecutively included in the test-group. Patients were eligible to participate if they had an indication for a bone-anchored hearing implant, were 18 years or older, and had no mental disability or psychiatric disease in medical history. We excluded patients with a bone thickness at implant site of less than $4 \mathrm{~mm}$, softtissue thickness of more than $10 \mathrm{~mm}$ or inability to show up on all follow-up visits. We additionally excluded patients with diseases or treatments known to compromise bone quality at the implant site (e.g., radiotherapy, osteoporosis, diabetes mellitus).

The last 25 patients implanted with a wide implant using the linear incision technique with tissue reduction in a randomized controlled trial comparing wide implants with previous generation implants were asked to participate as controls in the current study (5). Identical implants and abutments were used in both groups and surgeries were performed by the same surgeons. Additionally, the same eligibility criteria were adhered to, with exception of the maximum soft-tissue thickness criterion.

\section{Surgical Techniques and Follow-up}

All patients were implanted with the Ponto wide implant (diameter, $4.5 \mathrm{~mm}$; length, $4 \mathrm{~mm}$, Oticon Medical AB, Askin, Sweden) during single-staged surgery. Abutment length was determined on soft-tissue thickness for the test-group (0.5$3 \mathrm{~mm}$ : $6 \mathrm{~mm}$ abutment, $3-6 \mathrm{~mm}$ : $9 \mathrm{~mm}$ abutment, $6-10 \mathrm{~mm}$ : $12 \mathrm{~mm}$ abutment). Follow-up visits were scheduled at 7 days, 21 days (sound processor fitting), 12 weeks, and 6 months. In the control-group, the standard linear incision technique, including tissue reduction and placement of a $6 \mathrm{~mm}$ abutment, was applied in all patients (1). Follow-up visits were scheduled at the same time points as the tissue preservation cohort, with additional follow-up at 14 days, 28 days, 6 weeks, and 12 months. Extra assessments, intended for the current study, were included at the 12-month follow-up visit for control patients.

\section{Outcome Measures}

\section{Primary Outcome}

The primary outcome measure was numbness around the abutment. Numbness was assessed with a broken wooden cotton swab; gnostic (cotton side) and vital (broken, sharp wooden side) sensibility were determined at six selected locations (Fig. 1A) and percentages of correct answers were calculated. Subjective sensibility was additionally measured with a Visual Analogue Scale (VAS) from 0, no complaints, to 10, maximum numbness. Patients reported the area of
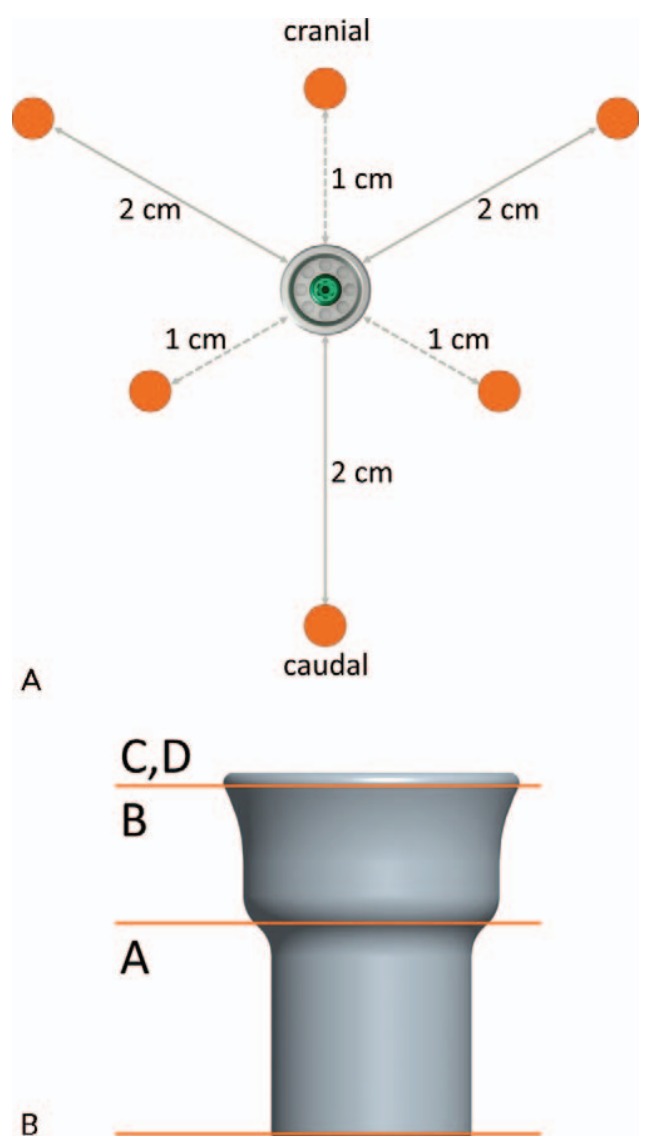

FIG. 1. A, Sensibility measurement locations, at all locations both gnostic (cotton side of wooden cotton swab) and vital sensibility (broken, sharp wooden side) will be tested randomly, the percentage correct answers were calculated for both and an overall percentage. $B$, Soft-tissue height relative to abutment (A under the shoulder of the abutment, B above the shoulder of the abutment, C partial overgrowth, D complete overgrowth).

subjective numbness as the diameter (centimeter) of skin with sensibility loss around the implant. Superiority of the tissue preservation technique was expected for the primary outcome variable.

\section{Secondary Outcomes}

Secondary outcomes included length of surgery measured from start of incision to end of surgery collected from the electronic patient file. Surgery times were excluded when start or end of surgery was missing, or when a bilateral procedure or an additional procedure was executed in the same setting.

Soft-tissue reactions were recorded according to Holgers' classification (15). The Holgers' soft classification is scored on a five-point scale from 0 , no signs of soft-tissue reaction, to 4 , an infection for which removal of implant is needed. Holgers grade 2 or higher was considered an adverse soft-tissue reaction in need of (local) treatment. Revision surgery (soft-tissue revision or abutment replacement) was recorded and skin height was assessed during follow-up. Skin height was related to the abutment using four different categories as shown in Figure 1B.

Scar assessment with the Patient and Observer Scar Assessment Scale (POSAS) v2.0 was conducted (16). The POSAS consists of a patient and an observer scale. Both scales contain 
six items with response options 1 to 10 . Response option 1 corresponds to normal skin and 10 to worst imaginable. The total score range is from 6 to 60 for both scales. The patient and the observer additionally score their overall opinion (not included in the total scores).

Implant loss was recorded during follow-up and Implant Stability Quotient (ISQ) was measured at all visits using resonance frequency analysis with the Osstell Mentor and a SmartPeg (type 55) (Osstell AB, Göteborg, Sweden). ISQ scores range from 0 to 100 , with increasing scores displaying a more rigid implant-bone interface, but the score is also a representation of implant variables such as implant diameter and abutment length (17). Longer abutments will result in lower absolute ISQ scores at baseline, so in the current study, trends are compared between different abutment lengths (18). Perpendicular measurements of the implant resulted in an ISQ high and an ISQ low value.

Furthermore, audiometric thresholds were collected. Bone conduction (BC) in situ thresholds were measured with the patients' sound processor on abutment and on a softband, additionally, BC thresholds were measured with a B71 transducer and audiometer (all measurements at a position $2 \mathrm{~cm}$ cranial of the abutment).

Subjective benefit was measured by the Glasgow Benefit Inventory (GBI), the Glasgow Health Status Inventory (GHSI), the Abbreviated Profile of Hearing Aid Benefit (APHAB) questionnaires $(19,20)$. The GBI is an 18-item questionnaire, which evaluates the patients' perceived benefit from an otorhinolaryngology intervention (19). Response options are on a five-point Likert scale ranging from large improvement to large deterioration in health status. Total scores range from - 100 (maximal adverse effect), 0 (no effect), to 100 (maximal positive effect). The GHSI measures the effect of a health problem on the quality of life of a person at the time the questionnaire is completed. The questionnaire contains 18 items and response options on a five-point Likert scale. Total scoring is from 0 to +100 , with higher scores indicating better health status. The APHAB is a 24-item inventory, scored in four 6-item subscales on communication abilities or perception of sound in daily life situations (20). All items are scored on a seven-point scale indicating frequency of problems experienced. An average unaided an aided score is calculated ranging from 1 to 99 , with higher scores indicating more problems.

The numbness assessment, POSAS scale, and audiometric thresholds were collected in the test-group at 6 months and in the control-group at 12 months. This was required because control patients already passed their 6 months visit before start of the current study. All other outcome measures were collected at the same time points in the test and the control-group. Outcome assessment was not blinded.

\section{Sample Size and Statistical Analysis}

No sample size calculations were made; investigators' experience and practically feasible number of patients determined the sample size. For comparison between test-group and the control-group Fishers nonparametric permutation test was used for numbness variables, Mann-Whitney $U$ test was used for other continuous variables, Mantel-Haenszel $\chi^{2}$ test for ordered categorical variables and Fisher's exact test for dichotomous variables. For analysis over time Wilcoxon Signed rank test was used for continuous variables. Nonparametric methods were chosen on non-normality assumptions and small sample sizes. Missing values were not imputed and no adjustments were made for multiple testing. In addition to the intention to treat analysis, we also performed a post-hoc per-protocol analysis on numbness and POSAS, excluding a patient in the control-group who had previous bone-implant surgery with tissue reduction. The test and control-groups had a different number of visits, thus for analysis including visit-based data, only data from follow-up visits available for both groups were included, for cumulative variables all visits including extra visits were included. All tests were two-tailed with significance levels of 0.05. Analyses were performed using SAS v9.4 (Cary, NC, U.S.A.). Data management and statistical analysis were performed by external data managers and biostatisticians (Statistiska Konsultgruppen, Göteborg, Sweden) according to a predefined statistical analysis plan.

\section{Ethical Considerations}

The clinical investigation was performed in accordance with the current version of the declaration of Helsinki (Washington 2002, ISO 14155) and was approved by the local ethical committee. The current study was registered with ClinicalTrials.gov and assigned the identifier NCT02064478.

\section{RESULTS}

\section{Patient and Surgery Characteristics}

All surgeries were performed between February 2014 and August 2014 in the test-group, and between March 2013 and January 2014 in the control-group. No patients were excluded in the test-group because of the additional skin thickness criterion. Baseline characteristics were similar between the two study groups (Table 1).

\section{Numbness Assessment}

The cotton swab numbness assessment resulted in a mean percentage of correct responses of 98\% (SD 4.4) and $89 \%$ (SD 15.0) in the test and control group

TABLE 1. Baseline characteristics ${ }^{a}$

\begin{tabular}{lcc}
\hline Characteristics & $\begin{array}{c}\text { Preservation } \\
\text { Group }(\mathrm{n}=25)\end{array}$ & $\begin{array}{c}\text { Reduction } \\
\text { Group }(\mathrm{n}=25)\end{array}$ \\
\hline Sex, $\mathrm{n}(\%)$ & $15(60 \%)$ & $10(40 \%)$ \\
$\quad$ Male & $10(40 \%)$ & $15(60.0)$ \\
Female & $51.5(13.4)$ & $53.9(12.2)$ \\
Age, yr, mean (SD) & $4(16 \%)$ & $4(16 \%)$ \\
Smoking at baseline, $\mathrm{n}(\%)$ & $26.0(3.9)$ & $26.5(4.2)$ \\
BMI at baseline, kg/m ${ }^{2}$, & $1(4 \%)$ & $3(12 \%)$ \\
mean (SD) & & \\
Skin disease at baseline, n (\%) & $21(84 \%)$ & $18(72 \%)$ \\
Indication, n (\%) & $1(4 \%)$ & $0(0 \%)$ \\
Acquired conductive/mixed & $3(12 \%)$ & $7(28 \%)$ \\
Congenital conductive & & \\
SSD & $0(0 \%)$ & $25(100 \%)$ \\
Abutment length selected, n (\%) & $17(68 \%)$ & $0(0 \%)$ \\
6 mm & $8(32 \%)$ & $0(0 \%)$ \\
$9 \mathrm{~mm}$ & & \\
$12 \mathrm{~mm}$ &
\end{tabular}

${ }^{\mathrm{a}}$ There were no significant differences in baseline characteristics between the two study groups. 
TABLE 2. Primary and secondary outcome measures

\begin{tabular}{|c|c|c|c|c|}
\hline Outcome & & $\begin{array}{l}\text { Preservation Group } \\
(\mathrm{n}=25) \text { Mean }(\mathrm{SD})\end{array}$ & $\begin{array}{l}\text { Reduction Group } \\
(\mathrm{n}=25) \text { Mean (SD) }\end{array}$ & $\begin{array}{c}p \\
\text { Value }\end{array}$ \\
\hline \multirow[t]{5}{*}{ Numbness at $6 / 12$ months $^{\mathrm{a}}$} & Gnostic sensibility, \% & $96.7(8.3)$ & $88.7(18.5)$ & 0.053 \\
\hline & Vital sensibility, $\%$ & $99.3(3.3)$ & $89.3(17.9)$ & 0.007 \\
\hline & Total sensibility, $\%$ & $98.0(4.4)$ & $89.0(15.0)$ & 0.003 \\
\hline & Subjective numbness, VAS & $0.36(1.10)$ & $1.69(2.44)$ & 0.051 \\
\hline & Area of subjective numbness, $\mathrm{cm}$ & $0.24(0.83)$ & $0.89(1.39)$ & 0.021 \\
\hline \multirow[t]{2}{*}{ Length of surgery, min } & Electronic patient file ${ }^{b}$ & $24.6(6.2)$ & $31.9(6.5)$ & $<0.001$ \\
\hline & Knife time & $20.8(4.3)$ & & \\
\hline \multirow{5}{*}{$\begin{array}{l}\text { Maximum Holgers across all visits } \\
0-6 \mathrm{~m},-\mathrm{n}(\%)\end{array}$} & Grade 0 & $11(44 \%)$ & $12(48 \%)$ & \\
\hline & Grade 1 & $7(28 \%)$ & $12(48 \%)$ & \\
\hline & Grade 2 & $4(16 \%)$ & $1(4 \%)$ & \\
\hline & Grade 3 & $3(12 \%)$ & $0(0 \%)$ & \\
\hline & Grade 4 & $0(0 \%)$ & $0(0 \%)$ & 0.14 \\
\hline \multirow[t]{2}{*}{ Mild versus adverse soft tissue reactions } & Grade $0-1$ & $18(72 \%)$ & $24(96 \%)$ & \\
\hline & Grade $2-4$ & $7(28 \%)$ & $1(4 \%)$ & 0.049 \\
\hline \multirow[t]{4}{*}{ Skin height at 6 months } & A-under shoulder & $18(72 \%)$ & $17(68 \%)$ & \\
\hline & B-above shoulder & $7(28 \%)$ & $8(32 \%)$ & \\
\hline & $\mathrm{C}$-partial overgrowth & $0(0 \%)$ & $0(0 \%)$ & \\
\hline & D_complete overgrowth & $0(0 \%)$ & $0(0 \%)$ & 1.00 \\
\hline \multirow[t]{16}{*}{ POSAS at $6 / 12$ months $^{c}$} & $\mathrm{P}$-pain & $2.72(1.77)$ & $2.44(1.96)$ & 0.41 \\
\hline & $\mathrm{P}$-itching & $2.48(2.10)$ & $2.84(2.53)$ & 0.79 \\
\hline & $\mathrm{P}-$ color & $2.88(2.15)$ & $3.88(2.51)$ & 0.14 \\
\hline & $\mathrm{P}$ —stiffness & $2.64(2.08)$ & $2.60(1.80)$ & 0.98 \\
\hline & $\mathrm{P}$-thickness & $2.76(2.70)$ & $3.48(2.20)$ & 0.067 \\
\hline & $\mathrm{P}$ _-irregularity & $2.36(2.25)$ & $3.64(2.20)$ & 0.017 \\
\hline & $\mathrm{P}$ _total score & $15.8(10.8)$ & $18.9(9.7)$ & 0.11 \\
\hline & $\mathrm{P}$ _-overall opinion & $2.44(2.31)$ & $3.36(1.87)$ & 0.014 \\
\hline & $\mathrm{O}$-vascularity & $2.88(1.30)$ & $3.64(1.25)$ & 0.010 \\
\hline & $\mathrm{O}$-pigmentation & $2.16(0.55)$ & $2.76(1.16)$ & 0.048 \\
\hline & $\mathrm{O}$-thickness & $2.92(1.63)$ & $3.32(1.63)$ & 0.26 \\
\hline & $\mathrm{O}$-relief & $2.84(1.28)$ & $3.56(1.53)$ & 0.048 \\
\hline & $\mathrm{O}$-pliability & $2.20(0.50)$ & $2.76(1.23)$ & 0.092 \\
\hline & $\mathrm{O}$-surface & $2.28(0.68)$ & $3.32(1.31)$ & $<0.001$ \\
\hline & $\mathrm{O}$-total score & $15.3(4.3)$ & $19.4(6.3)$ & 0.006 \\
\hline & $\mathrm{O}$-overall opinion & $2.84(1.21)$ & $3.76(1.30)$ & 0.006 \\
\hline \multirow[t]{3}{*}{ ISQ low } & Baseline & $54.4(3.5)$ & $61.0(3.4)$ & $<0.001$ \\
\hline & AUC $0-6$ months & $57.0(4.1)$ & $64.2(3.1)$ & $<0.001$ \\
\hline & Change in ISQ $0-6$ months & $+4.6(2.0)$ & $+4.4(3.2)$ & 0.86 \\
\hline \multirow[t]{5}{*}{$\mathrm{BC}$ in situ at $6 / 12$ months, $\mathrm{dB}$} & Abutment, $250 \mathrm{~Hz}$ to $8 \mathrm{kHz}$ & $25.5(12.8)$ & $25.0(11.8)$ & 0.93 \\
\hline & Testband, $250 \mathrm{~Hz}$ to $8 \mathrm{kHz}$ & $37.8(11.3)$ & $35.5(10.0)$ & 0.53 \\
\hline & $\mathrm{B} 71,250$ to $4 \mathrm{kHz}$ & $22.4(11.2)$ & $20.9(11.7)$ & 0.79 \\
\hline & Abutment-testband, $250 \mathrm{~Hz}$ to $8 \mathrm{kHz}$ & $-12.2(7.0)$ & $-10.5(4.7)$ & 0.10 \\
\hline & Abutment-B71, 250 to $4 \mathrm{kHz}$ & $-0.99(4.86)$ & $-0.48(4.72)$ & 0.79 \\
\hline
\end{tabular}

\footnotetext{
${ }^{\mathrm{a}}$ Intention to treat analysis is presented. Slightly different results are shown for per protocol analysis in the control group. Gnostic: mean control group 88.2 (SD, 18.7), $p=0.044$. Vital: $88.9(18.2), p=0.003$. Total: $88.5(15.1), p=0.002$. Subjective: $1.76(2.47), p=0.039$. Area of subjective: $0.93(1.41), p=0.016$.

$\mathrm{b}_{\mathrm{n}}=20$ for test group and $\mathrm{n}=22$ for control group.

${ }^{\mathrm{c}}$ Intention to treat analysis is presented. Slightly different results are shown for per protocol analysis in the control group. Largest differences were seen for the patient scale on stiffness (mean $2.46(\mathrm{SD}, 1.69), p=0.87)$, and for the observer scale on pliability $(2.63(1.06), p=0.14)$, surface $(3.21(1.22), p=0.002)$, and relief (3.46 (1.47), $p=0.071)$. On total scores this resulted in 18.5 (9.6) for the patient scale and 19.0 (6.2) for the observer scale.

ISQ indicates Implant Stability Quotient; POSAS, Patient and Observer Scar Assessment Scale.
}

respectively ( $p=0.003)$. The subjective numbness assessment with the VAS score resulted in a mean VAS of 0.4 (SD 1.1) in the test-group and 1.7 (SD 2.4) in the controlgroup $(p=0.051)$. The per protocol analysis showed similar results for all comparisons (Table 2).

\section{Length of Surgery}

The mean surgical time as registered in the electronic patient file was 24.6 minutes (SD, 6.2; range, 13-39) in the test-group and 31.9 minutes (SD, 6.5; range, 20-44) for the control-group $(p<0.001)$. In the test-group the 
length of surgery was also recorded as knife time only (measured by the investigator), resulting in 20.8 minutes (SD, 4.3; range, 13-29).

\section{Adverse Events}

In the test-group device/surgery-related adverse events included one patient with fever in the first days postoperative without any local signs of infection, one patient with persistent itch around the abutment, and one patient with ongoing pain around the abutment (not able to get sound processor on abutment, different sound processor selected). In the control-group related events included two patients with a wound dehiscence at 7 days, two patients with mild pain around the abutment, one patient with scar hypertrophy, and one patient with an abscess next to the abutment (required recurrent incision and antibiotic treatment).

For the test-group seven patients required one additional unplanned visit, in the control-group two patients required one unplanned visit, and one patient required four unplanned visits (patient with abscess).

\section{Other Soft-tissue Outcomes}

\section{Soft-tissue Reactions}

In both groups no Holgers grade 4 reactions were recorded. Comparing all Holgers grades as maximum per implant across all visits between groups, no significant difference was found ( $p=0.14)$. When comparing clinically relevant adverse reactions (Holgers $\geq 2$ ), $28 \%$ $(n=7)$ adverse reactions in the test-group versus $4 \%$ $(\mathrm{n}=1)$ in the control-group were observed $(p=0.049)$. The rate of adverse soft-tissue reactions per visit was $7.5 \%$ ( $\mathrm{n}=8$ in 282 visits, one patient with two adverse soft-tissue reactions) and $0.6 \%(\mathrm{n}=1$ in 356 visits $)$. All adverse soft-tissue reactions resolved after one or two local treatment regimens.

\section{Revision Surgery and Skin Height}

One revision surgery was performed in the controlgroup 1 month after initial surgery because of high skin. No partial or complete soft-tissue overgrowth was observed, and no differences between groups were noticed in skin height during first 6 months.

\section{POSAS}

On the patient scale highest values were scored for color, thickness, and irregularity. Differences between groups were noted on irregularity and on overall opinion. The mean total score for the patient scale was 15.8 (SD 10.8 ) for the test-group versus 18.9 (SD 9.7) for the control-group $(p=0.11)$. On the observer scale highest values were scored for vascularity, thickness, and relief. Differences between groups were noticed in vascularity, pigmentation, surface, and overall opinion. The mean total observer score was 15.3 (SD 4.3) for the test-group versus 19.4 (SD 6.3) for the control-group ( $p=0.006)$. The per protocol analysis showed similar results (Table 2).

\section{Implant Loss and ISQ}

No implants were lost in either group. ISQ low was higher at baseline (surgery) in the control-group $(p<0.001)$, as was expected with longer abutments in the test-group, and remained higher during follow-up (AUC ISQ low $0-6 \mathrm{mo}, p<0.001$ ). Nevertheless, no difference between groups was recorded in the change of ISQ from surgery. ISQ low showed a mean increase of $+4.6(\mathrm{SD} 2.0)$ in the test-group and +4.4 (SD 3.2) in the control-group (change within groups $p<0.001$; difference between groups $p=0.86$ ). ISQ high showed similar results, with absolute numbers 1 to 2 points higher on average and slightly less increase over time (Fig. 2B).

\section{Audiology}

The overall thresholds at $250 \mathrm{~Hz}$ to $8 \mathrm{kHz}$ on testband and at $250 \mathrm{~Hz}$ to $4 \mathrm{kHz}$ on B71 showed no differences between groups, indicating similar hearing thresholds. The overall thresholds on abutment, which are clinically most relevant given similar hearing thresholds, showed no difference between groups (mean 25.5 [SD 12.8] versus 25.0 [SD 11.8], $p=0.93$ ). Additionally, the overall difference between abutment and testband $(250 \mathrm{~Hz}$ to $8 \mathrm{kHz}$ ) showed no difference between test and controlgroup (mean -12.2 [SD 7.0] versus - 10.5 [SD 4.7], $p=0.10)$.

Comparing individual frequency thresholds between the two study groups for the abutment and for the B71 conditions, no significant differences where noticed. In the testband condition a difference was noted at the $1-\mathrm{kHz}$ frequency (mean, 30.2 [SD 12.9] versus 20.8 [SD 14.0], $p=0.028$ ). Additionally, comparisons between different conditions on individual frequencies were performed. For comparison between test and control-group when looking at the difference between abutment and testband, a significant difference was noticed at $1 \mathrm{kHz}$ (mean -14.4 [SD 7.8] versus -5.0 [SD 7.2], $p<0.001$ ) and $1.5-\mathrm{kHz}$ frequency (mean -9.6 [SD 7.1] versus -5.4 [SD 6.8], $p=0.037$ ). When comparing abutment to $\mathrm{B} 71$ conditions, a difference was observed on the $1.5-\mathrm{kHz}$ situation (mean -5.8 [SD 5.5] versus -2.0 [SD 7.8], $p=0.044$ ). All mean thresholds per frequency are shown in Table 3.

\section{Health-related Quality of Life}

The GBI was completed 12 weeks after surgery. The GBI showed a positive result $(>0)$ in $98 \%$ of all 50 patients on the total score (mean total score 32.3, SD 19.9 , range -6.3 to 88.9 ). On the general subscale $96 \%$ of patients scored positively. The social and physical subscale showed a neutral effect in most patients, 75\% respectively $74 \%$, and a considerable proportion with a positive result, $25 \%$ respectively $18 \%$.

The GHSI and APHAB were both assessed at 6 months. For the GHSI total score baseline (best score, either aided or unaided) to visit at month 6 (aided) a significant mean improvement of +12.4 (SD 10.5; $p<0.0001)$ was observed. For the general score also an improvement was observed, the social and physical scores did not show an improvement or deterioration. For 


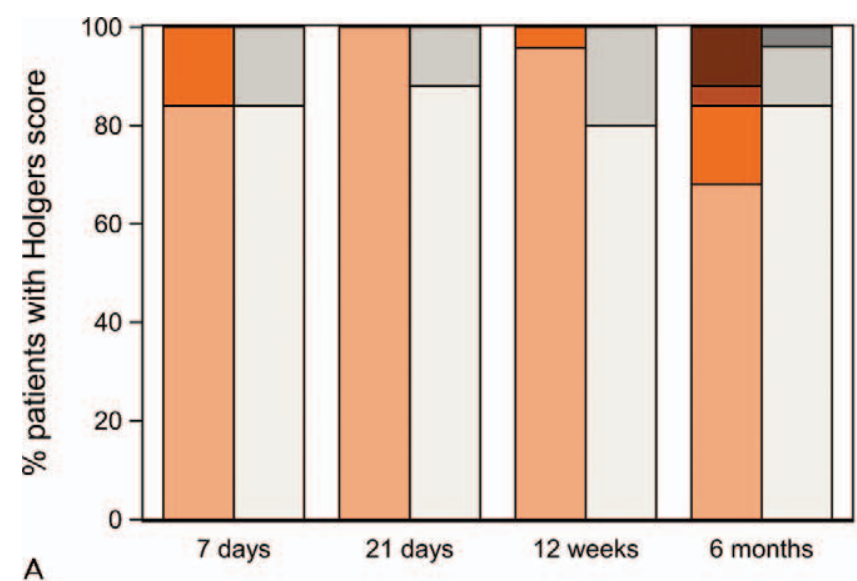

Test Control

Holgers 0

Holgers 1

Holgers 2

Holgers 3
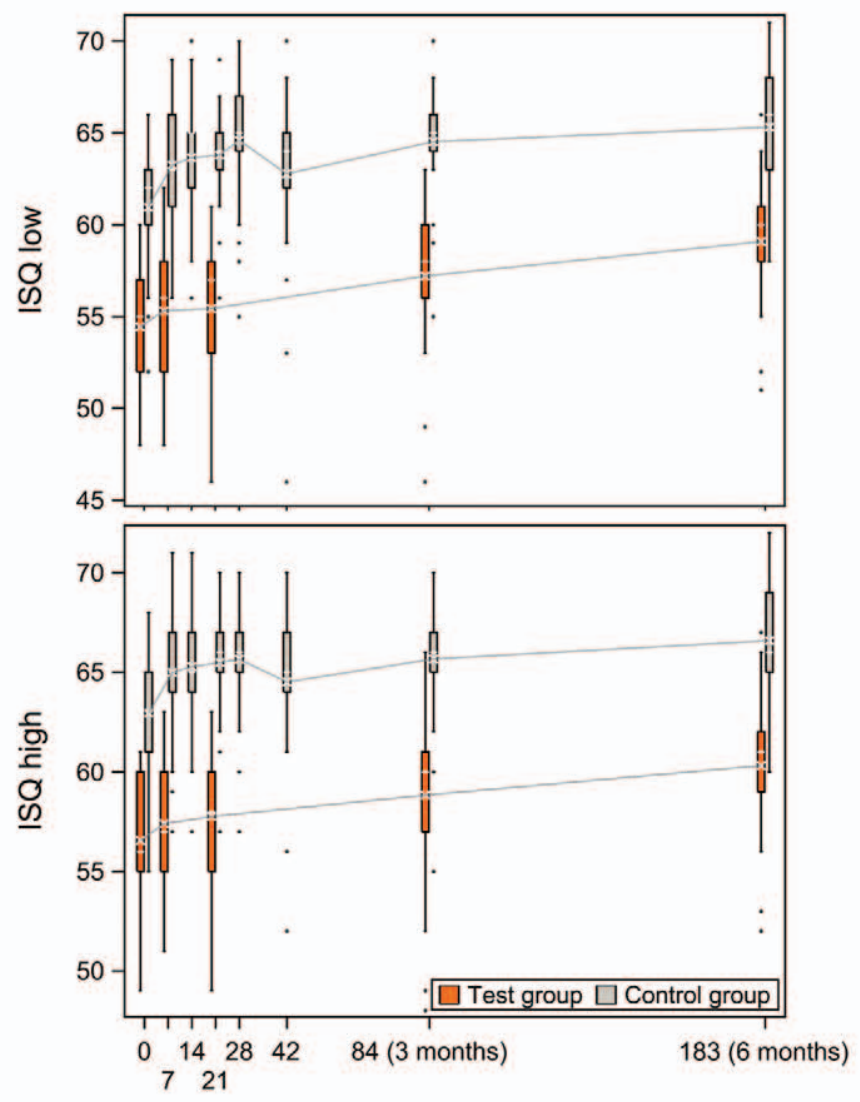

B

Days since surgery

FIG. 2. A, Soft-tissue reactions according to Holgers' classification, as a percentage per visit per study group, not including extra visits. $B$, Box-and-whisker plots of ISQ low and ISQ high measurements over time. The mean (cross) and median (horizontal bar) are defined within each plot, boxes represent interquartile range, whiskers represent $95 \%$ range, and dots represent outlier values. ISQ indicates Implant Stability Quotient.

the APHAB questionnaire an improvement was noted on the mean total score at 6 months compared with best baseline score of -24.3 (SD 23.1; $p<0.0001$ ). This difference was observed on all subscales with exception of the aversiveness scale (Fig. 3).

No significant differences were found between test and control-group in GBI results or benefit on the GHSI and $\mathrm{APHAB}$.

\section{DISCUSSION}

\section{Key Findings}

Tissue preservation surgery resulted in better results on sensibility and POSAS scar assessment scale and shorter surgery time. However, significantly more adverse softtissue reactions according to the Holgers score were observed. For ISQ and audiology the study did not 


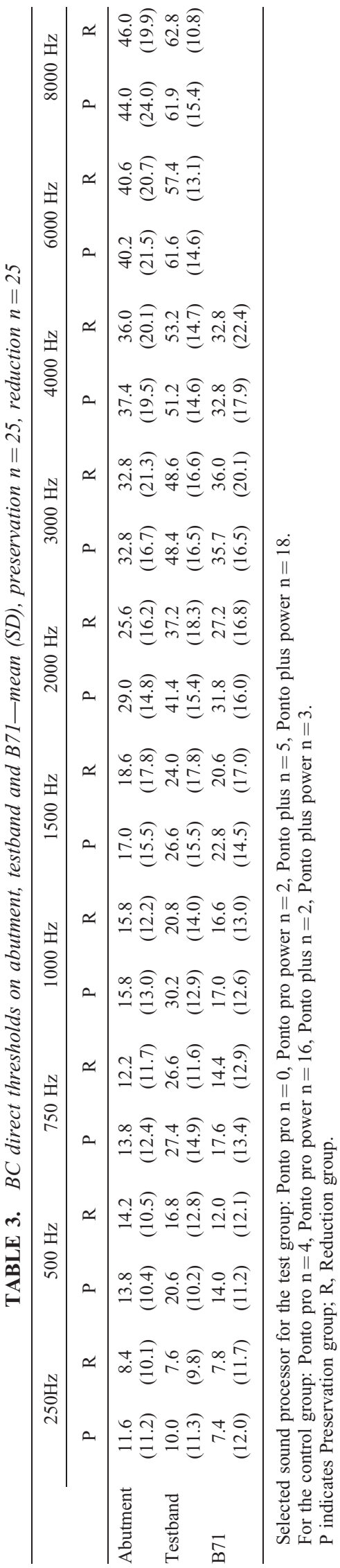

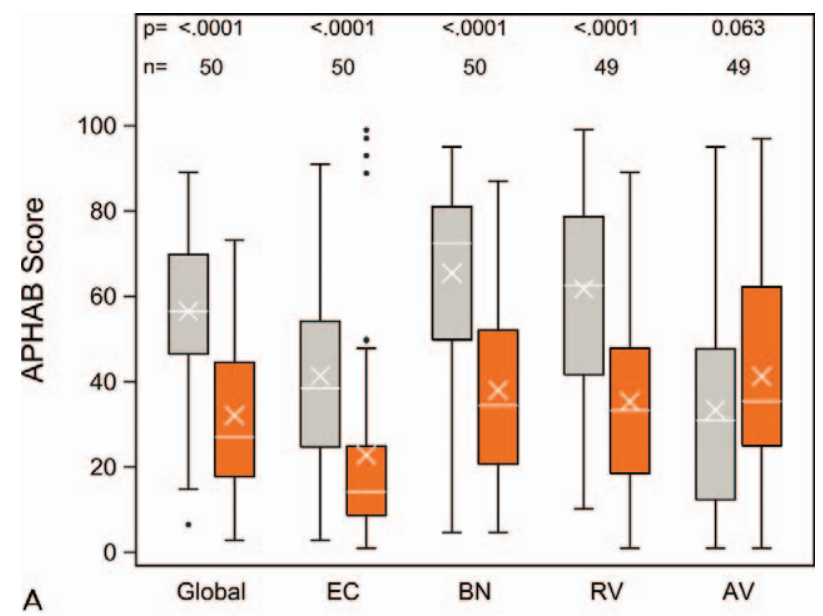

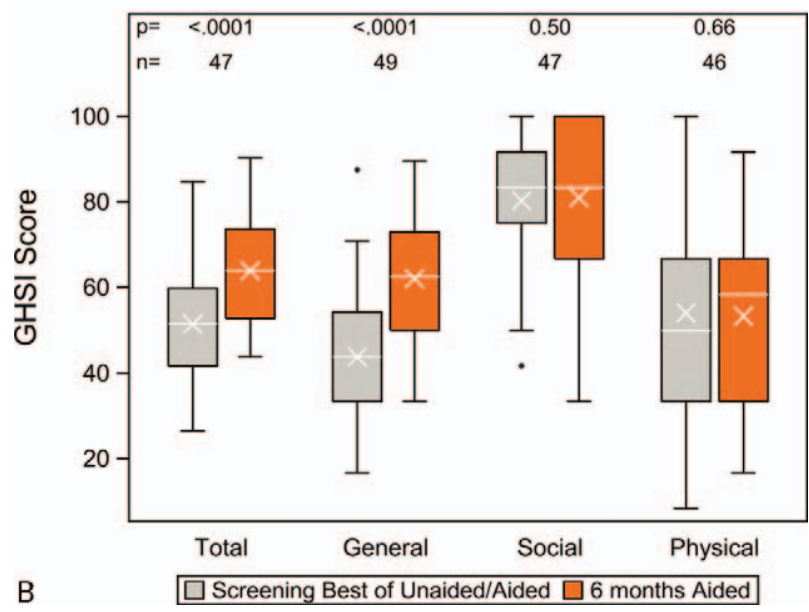

FIG. 3. Health-related quality of life measured by the GHSI and APHAB questionnaires, completed before surgery (best score, either aided or unaided) and after 6 months (aided) (APHAB indicates Abbreviated Profile of Hearing Aid Benefit; AV, aversiveness; BN, background noise; EC, ease of communication; GHSI, Glasgow Health Status Inventory; RV, reverberation). The mean (cross) and median (horizontal bar) are defined within each plot, boxes represent interquartile range, whiskers represent $95 \%$ range, and dots represent outlier values.

provide evidence that tissue preservation is better or worse compared with tissue reduction.

\section{Strengths and Limitations}

One of the important strengths of the current study is the selection of the control-group with a standard linear incision with tissue reduction. All studies performed so far use no or a less ideal control-group; consequently, superiority of the new technique over the current gold standard could not be concluded on these studies $(6-9,12)$. Aside from the selection of the control-group, the current study adds important results on audiological outcomes.

By taking the control patients from a previous trial, the interventions could not be randomized and follow-up visits were separated in time for the two study groups. 
The 12-month evaluation of the control-group was, however, expected to result in better outcomes on sensibility and POSAS for the control-group, since healing of softtissue would be more complete at longer follow-up. We feel that the benefit of fewer patients needed for participation and earlier availability of results outweigh the drawbacks of the selected study design. Moreover, both groups were included with the same eligibility criteria and had comparable baseline characteristics.

Follow-up visits were more tightly spaced in the control-group. It might be possible that more frequent visits to the out-patient clinic result in better soft-tissue care. Conversely, more than half of adverse soft-tissue reactions were noticed at 6 months and the more tightly spaced visits were completed in the first 6 weeks.

Another limitation could be the nonblinded follow-up. However, since both surgical techniques result in different appearance of implant sites and abutment lengths, with accompanying ISQ values, blinding of observers and patients would not be feasible.

\section{INTERPRETATION}

For the primary outcome variable numbness, we recorded significant differences in favor of the tissue preservation technique, though in most patients treated with standard linear incision technique also good sensibility scores of $>90 \%$, and low VAS scores with small areas of subjective numbness were observed. Additionally, several patients reported difficulties answering the questions on this scale because of limited visibility, especially on the color and overall question. Moreover, patients reported limited importance of both VAS and area of numbness and appearance of the scar behind the ear during evaluation with the POSAS. This might though be biased by the short follow-up and dominated patients' perspectives by the good outcomes on hearing improvement.

The difference in absolute ISQ scores at baseline and during follow-up can be explained by different abutment lengths (18). Since trends in ISQ during follow-up showed a similar development, no evidence of stability differences is suggested by these data. The increased soft tissue reactions should be interpreted with care, and further follow-up is needed to draw conclusions. The number of adverse soft-tissue reactions in the controlgroup ( $\mathrm{n}=1,4 \%)$, was lower compared with the percentage of adverse soft-tissue reactions in the complete study group from which the current control-group was a subset (5). A pathophysiological hypothesis for the increased soft-tissue reactions could be the result of more free movement of soft-tissue surrounding the abutment, as was described previously by Brånemark and Albrektsson (21).

All implants were loaded with a sound processor 3 weeks after surgery. The current good results suggest that loading at 3 weeks seems to be safe at short-term follow-up, also when using tissue preservation and longer abutments. The literature supports these early loading protocols with even longer follow-up and similar good outcomes in the standard linear incision technique $(22,23)$.

Comparing audiological outcomes by $\mathrm{BC}$ in situ measurements, we noticed only minor differences in thresholds between both groups, except for testband at $1 \mathrm{kHz}$ and difference between abutment-B71 at $1.5 \mathrm{kHz}$ and abutment-testband on 1 and $1.5 \mathrm{kHz}$. The significantly poorer results on these frequencies for the testgroup may be because of changes in resonance frequency of the sound processor in the transcutaneous conditions, resulting in less output in the mid frequencies (14). This effect is most strongly present in patients with tissue preservation. Nevertheless, it seems that surrounding skin around abutments and longer abutments did not affect the transmission of sound at 6-month follow-up with current set-up.

\section{Generalizability and Conclusions}

This study adds knowledge in the rapidly evolving tissue preservation trend in bone-anchored hearing implant surgery. Since we noticed some conflicting results compared with the existing literature, like the increase in soft-tissue reactions according to Holgers, this study emphasizes the need for a more elaborate evaluation of new surgical techniques before complete substitution of currently applied methods is sensible.

On the basis of the current 6-month results, the overall difference between these surgical techniques from a patient's perspective might not be evidently in favor of either tissue preservation or tissue reduction: comparable results on audiology, less numbness, and better POSAS scores, yet more soft-tissue reactions according to Holgers were recorded in the first 6 months. However, since adverse soft-tissue reactions in the study completely recovered after one or two local treatment regimens, the burden for patients might be relatively minor. An additional important gain is the reduction of surgery times.

Longer follow-up of the current study will be needed to draw firm conclusions, especially regarding soft-tissue reactions. Data on 36-month results will be reported for this study later.

Acknowledgments: The authors thank Teja Repkes and Mieki Verbruggen for contributions in audiological data collection.

\section{REFERENCES}

1. de Wolf MJF, Hol MKS, Huygen PLM, Mylanus EAM, Cremers CWRJ. Clinical outcome of the simplified surgical technique for BAHA implantation. Otol Neurotol 2008;29:1100-8.

2. Mohamad S, Khan I, Hey SY, Hussain SSM. A systematic review on skin complications of bone-anchored hearing aids in relation to surgical techniques. Eur Arch Otorhinolaryngol 2016;273: $559-65$.

3. van de Berg R, Stokroos RJ, Hof JR, Chenault MN. Bone-anchored hearing aid: A comparison of surgical techniques. Otol Neurotol 2010;31:129-35.

4. Nelissen RC, Stalfors J, de Wolf MJF, et al. Long-term stability, survival, and tolerability of a novel osseointegrated implant for bone conduction hearing: 3-year data from a multicenter, randomized, controlled, clinical investigation. Otol Neurotol 2014;35: $1486-91$. 
5. Nelissen RC, Besten den CA, Mylanus EAM, Hol MKS. Stability, survival, and tolerability of a $4.5-\mathrm{mm}$-wide bone-anchored hearing implant: 6-month data from a randomized controlled clinical trial. Eur Arch Otorhinolaryngol 2016;273:105-11.

6. Hultcrantz M. Outcome of the bone-anchored hearing aid procedure without skin thinning: A prospective clinical trial. Otol Neurotol 2011;32:1134-9.

7. Hultcrantz M, Lanis A. A five-year follow-up on the osseointegration of bone-anchored hearing device implantation without tissue reduction. Otol Neurotol 2014;35:1480-5.

8. Høgsbro M, Agger A, Johansen LV. Bone-anchored hearing implant surgery: Randomized trial of dermatome versus linear incision without soft tissue reduction-clinical measures. Otol Neurotol 2015;36:805-11.

9. Singam S, Williams R, Saxby C, Houlihan FP. Percutaneous boneanchored hearing implant surgery without soft-tissue reduction: Up to 42 months of follow-up. Otol Neurotol 2014;35:1596-600.

10. Hawley K, Haberkamp TJ. Osseointegrated hearing implant surgery: Outcomes using a minimal soft tissue removal technique. Otolaryngol Head Neck Surg 2013;148:653-7.

11. Husseman J, Szudek J, Monksfield P, Power D, O’Leary S, Briggs R. Simplified bone-anchored hearing aid insertion using a linear incision without soft tissue reduction. J Laryngol Otol 2013;127 (Suppl 2S2):S33-8.

12. Hultcrantz M. Stability testing of a wide bone-anchored device after surgery without skin thinning. Biomed Res Int 2015;2015:853072-6.

13. Gordon SA, Coelho DH. Minimally invasive surgery for osseointegrated auditory implants: A comparison of linear versus punch techniques. Otolaryngol Head Neck Surg 2015;152:1089-93.

14. Tjellström A, Håkansson $B$, Lindström J, et al. Analysis of the mechanical impedance of bone-anchored hearing aids. Acta Otolaryngol 1980;89:85-92.
15. Holgers KM, Tjellström A, Bjursten LM, Erlandsson BE. Soft tissue reactions around percutaneous implants: A clinical study of soft tissue conditions around skin-penetrating titanium implants for bone-anchored hearing aids. Am J Otol 1988;9:56-9.

16. van de Kar AL, Corion LUM, Smeulders MJC, Draaijers LJ, van der Horst CMAM, van Zuijlen PPM. Reliable and feasible evaluation of linear scars by the Patient and Observer Scar Assessment Scale. Plast Reconstr Surg 2005;116:514-22.

17. Sennerby L, Meredith N. Implant stability measurements using resonance frequency analysis: Biological and biomechanical aspects and clinical implications. Periodontol 2000 2008;47:51-66.

18. Nelissen RC, Wigren S, Flynn MC, Meijer GJ, Mylanus EAM, Hol MKS. Application and interpretation of resonance frequency analysis in auditory osseointegrated implants: A review of literature and establishment of practical recommendations. Otol Neurotol 2015;36:1518-24.

19. Robinson K, Gatehouse S, Browning GG. Measuring patient benefit from otorhinolaryngological surgery and therapy. Ann Otol Rhinol Laryngol 1996;105:415-22.

20. Cox RM, Alexander GC. The abbreviated profile of hearing aid benefit. Ear Hear 1995;16:176-86.

21. Brånemark PI, Albrektsson T. Titanium implants permanently penetrating human skin. Scand J Plast Reconstr Surg 1982;16: $17-21$.

22. Faber HT, Dun CAJ, Nelissen RC, Mylanus EAM, Cremers CWRJ, Hol MKS. Bone-anchored hearing implant loading at 3 weeks: stability and tolerability after 6 months. Otol Neurotol 2013;34: 104-10.

23. Nelissen RC, Besten den CA, Faber HT, Dun CAJ, Mylanus EAM, Hol MKS. Loading of osseointegrated implants for bone conduction hearing at 3 weeks: 3 -year stability, survival, and tolerability. Eur Arch Otorhinolaryngol 2015 [Epub ahead of print] 